\title{
Generation of $J_{0}$-Bessel-Gauss beam by a heterogeneous refractive index map
}

\author{
Damian P. San-Roman-Alerigi, ${ }^{1}$ Tien K. Ng, ${ }^{1}$ Yaping Zhang, ${ }^{1}$ Ahmed Ben Slimane, \\ Mohammad Alsunaidi, ${ }^{1,2}$ and Boon S. Ooi ${ }^{1, *}$ \\ ${ }^{1}$ Photonics Laboratory, Division of Physical Sciences and Engineering, King Abdullah University of Science and \\ Technology (KAUST), Saudi Arabia \\ ${ }^{2}$ King Fahd University of Petroleum and Minerals (KFUPM), Saudi Arabia \\ *Corresponding author: boon.ooi@kaust.edu.sa
}

Received January 17, 2012; revised March 13, 2012; accepted March 14, 2012; posted March 15, 2012 (Doc. ID 161710); published June 6, 2012

\begin{abstract}
In this paper, we present the theoretical studies of a refractive index map to implement a Gauss to a $J_{0}$-BesselGauss convertor. We theoretically demonstrate the viability of a device that could be fabricated on a $\mathrm{Si} / \mathrm{Si}_{1-y} \mathrm{O}_{y} / \mathrm{Si}_{1-x-y} \mathrm{Ge}_{x} \mathrm{C}_{y}$ platform or by photo-refractive media. The proposed device is $200 \mu \mathrm{m}$ in length and $25 \mu \mathrm{m}$ in width, and its refractive index varies in controllable steps across the light propagation and transversal directions. The computed conversion efficiency and loss are $90 \%$, and $-0.457 \mathrm{~dB}$, respectively. The theoretical results, obtained from the beam conversion efficiency, self-regeneration, and propagation through an opaque obstruction, demonstrate that a two-dimensional (2D) graded index map of the refractive index can be used to transform a Gauss beam into a $J_{0}$-Bessel-Gauss beam. To the best of our knowledge, this is the first demonstration of such beam transformation by means of a $2 \mathrm{D}$ index-mapping that is fully integrable in silicon photonics based planar lightwave circuits (PLCs). The concept device is significant for the eventual development of a new array of technologies, such as micro optical tweezers, optical traps, beam reshaping and nonlinear beam diode lasers. (C) 2012 Optical Society of America
\end{abstract}

OCIS codes: $\quad 050.1940,290.3200,260.1960$

\section{INTRODUCTION}

In 1987, Durnin introduced nondiffracting $J_{p}$ Bessel beams as members of a family of solutions to the homogeneous Helmholtz equation, with distinctive properties; e.g., their transverse profile does not change as the beam propagates in free space and exhibit self-regeneration when disturbed by nontransparent obstacles [1]. Among them the zero order, $J_{0}$, Bessel beam has captured the most attention due to its peculiar intensity distribution, which is focused on the propagation axis, besides nondiffracting propagation [2].

While mathematically viable, Bessel beams are not square integrable; i.e., the beam contains infinite energy, thus rendering the ideal solution infeasible [3,4]. Yet good approximation exists, namely Bessel-Gauss beams. Introduced by Gori et al. [4], these solutions to Helmholtz homogeneous equations resemble the ideal $J_{p}$ Bessel beam with the sole difference that they bear finite power, and, therefore, can be realized experimentally, while retaining the primordial characteristics of self-reconstruction and diffraction-free propagation for lengths of interest to many optical applications, e.g., optical tweezers and microscopy [4-7].

Experimentally, Durnin $e \bar{t} \overline{a l}$. [8] were the first to show that it is possible to generate Bessel-Gauss beams by means of a circular slit and a lens placed one focal length away. Wu et al. [9] proposed a Nd:YAG cavity with a diffractive mirror to transform the Gauss beam from a pump laser into a BesselGauss beam of arbitrary order; Arrizón et al. [10] and Otero [11] independently used holograms to alter Gauss beams and produce zero and first order Bessel-Gauss beams; lately, Zhan [12] has shown that it is possible to generate them by using a radially polarized beam and surface plasmon resonance. Alternatively, Cong et al. [2] used phase elements to generate zero order Bessel-Gauss beams.

Recently, novel designs have used waveguides to produce diffraction-free beams, v.gr. Canning [13] presented a Fresnel waveguide as a diffraction-free mode generator, and Ilchenko et al. [14] experimentally showed that cylindrical waveguides can produce truncated Bessel-Gauss beams; also, Tsai et al. [15] showed that is possible to use an acoustic tunable lens to generate this family of beams.

The aforementioned methods produce high quality beams. Nonetheless, their design and fabrication on photonic integrated circuits (PICs) or planar lightwave circuits (PLCs) would involve complex processing steps, rendering the integration into most PIC platforms impractical. It is a matter of debate whether resonator/waveguide designs (see, for example, $[13,14])$ could be used for this purpose. In our experience on such devices, the quality of the beam, and conversion efficiency, critically depend on the coupling efficiency. Moreover, the converted beam free space propagation is limited to $\sim 1 \mathrm{~mm}$ [14]. Hence the motivation to attain a fully integrated Gauss to $\bar{J}_{0}$-Bessel-Gauss microconvertor based on current Si foundry technology is justified. If viable, it could be readily integrated into semiconductor-chip-based optical elements of great importance to a manifold of optical applications. For instance, they have been shown to increase the scanning resolution and tissue penetration depth of optical coherence tomography (OCT) systems by $\sim 50 \%$ [16]

\section{PROBLEM DESCRIPTION AND MODELING}

In Durnin's primordial design, an impinging Gauss beam diffracts from the surface of an axicon lens $[6,8,17]$, forming a 
converging wavefront and thus giving raise to the BesselGauss beam. Analytically, it can be described by means of diffraction at the Fresnel limit. However accurate this method could be, it presumes the outbound field to extent infinitely in the transversal direction in order to comply with the boundary conditions []. To avoid this complication, we will assume hereafter the output beam to be $J_{0}$-Bessel-Gauss as defined by Gori et al. [4], i.e., the superposition of Gauss and $J_{0}$-Bessel profiles.

It could be contended that only a PIC-integrable-axicon lens would suffice to achieve the desired conversion. Such a lens, though, is equivalent to the fabrication of a continuous alteration of the index of refraction, albeit homogeneous, over a conic volume, posing practical and nontrivial integration problems when it is manufactured using the well-established silicon fabrication technologies, namely the difficulty to shape the conic surface. To alleviate this conundrum, we seek a practical profile of the refractive index that can act as feasible substitute for the axicon lens, suited for integration into current silicon foundry and manufacturing technologies.

Before proceeding any further, it is important to remark that nonsingular graded refractive indexes (GRIN) cannot yield perfect nondiffracting beams as proven by Hayata [18]. However, a refractive index mapping can potentially achieve high quality conversion from a Gauss beam to a $J_{0}$-BesselGauss beam, which is different from the fundamental Bessel beam presented by Durnin et al. [1].

In Fig. 1, the input and output beams are known a priori, and hence the problem is reduced to determining the heterogeneous refractive index in region $A$, which will result in the transformation of the input to output fields in region $B$. This is the optical inverse scattering problem [19-22].

Analytically, the input and output fields are described by harmonic functions of the form $\vec{E}_{i}=\vec{\phi}(\vec{k}, \vec{r}) e^{i \omega t}$ and $\vec{E}_{o}=$ $\vec{\zeta}(\vec{k}, \vec{r}) e^{i \omega t}$, respectively; where $\vec{\phi}$ and $\vec{\zeta}$ are solutions to the homogeneous Helmholtz equation. In the transformation region, medium $A$, the field is $\vec{E}_{A}=\vec{\vartheta}(\vec{k}, \vec{r}) \tau(t)$, where $\vec{\vartheta}$ is determined by the optical properties of the medium. If the material is linear, then $\tau(t)=e^{i \omega t}$, and the problem narrows to determining the shape of the permittivity, $\varepsilon$, and permeability, $\mu$, that realize the transformation $\vec{\phi} \rightarrow \vec{\zeta}$ after some finite propagation distance, $d$.

In reality, the manipulation of permeability, $\mu$, is complex. On that account, the transformation medium $A$ is set to be nonmagnetic, with some constant value for $\mu$. Consequently, Maxwell's equation for the electric field $\vec{E}$ for this region is written as

$$
\nabla^{2} \vec{E}-\mu \varepsilon(\vec{r}) \frac{\partial^{2} \vec{E}}{\partial t^{2}}=-\nabla\left(\frac{1}{\varepsilon} \vec{E} \cdot \nabla \varepsilon\right)
$$

where $\varepsilon(\vec{r})$, the permittivity, is a real value function and $\vec{r}=$ $(u, v, w)$ describes a position vector in the transformation space. Outside the device, the material is homogeneous; i.e., $\varepsilon(\vec{r})=$ constant for all $\vec{r} \in B$, where both Gaussian and Bessel functions are well known solutions to Eq. (1). Inside the device, the permittivity is limited to the range achievable in practical fabrication methods:

$$
\varepsilon_{\min } \leq \varepsilon(\vec{r}) \leq \varepsilon_{\max }, \quad \forall \vec{r} \in A
$$

Recalling that if $E_{A}=\vec{\vartheta}(\vec{r}) \tau(t)$, then Eq. (1) can be reduced to the time independent Helmholtz equation,

$$
\nabla^{2} \vec{\vartheta}(\vec{r})-\omega^{2} \mu \varepsilon(\vec{r}) \vec{\vartheta}(\vec{r})=\nabla(\vec{\vartheta}(\vec{r}) \cdot \nabla \ln \varepsilon(\vec{r}))
$$

Observe that if the field $\vec{\vartheta}(\vec{r})$ is given, then the only unknown in Eq. (3) would be $\varepsilon(\vec{r})$, which is the permittivity map that we wish to determine. Henceforth let us examine $\vec{\vartheta}(\vec{r})$.

Denote by $S_{i}$ and $S_{o}$ the boundary surfaces between region $A$, and the incident $B_{i}$ and exit $B_{o}$ surfaces, respectively. Then $\vec{\vartheta}(\vec{r})$ can be split into the three regions:

$$
\vec{\vartheta}(\vec{r})=\left\{\begin{array}{lll}
\vec{\phi}(\vec{r}) & \forall & \vec{r} \in S_{i}, \\
\vec{\psi}(\vec{r}) & \forall & \vec{r} \in A, \\
\vec{\zeta}(\vec{r}) & \forall & \vec{r} \in S_{o},
\end{array}\right.
$$

where $\vec{\psi}: A \rightarrow R^{3}$ is the transforming field. Since we wish to control $\vec{\vartheta}$, and then decrease the complexity of the problem, we define the shape of $\vec{\psi}$ across region $A$. Accordingly, we introduce the real valued functions $f, g: A^{3} \rightarrow[0,1]$ and $\vec{\gamma}: A \rightarrow R^{3}, C^{2}$ continuous and square integrable, i.e., smooth functions. These functions are invoked to describe the transforming beam as a superposition of the desired input and output fields, in addition to an arbitrary field $\vec{\gamma}$ that can be thought to represent the residual beam. The field $\vec{\psi}(\vec{r})$ can be written now as

$$
\vec{\psi}(\vec{r})=f(\vec{r}) \vec{\phi}(\vec{r})+g(\vec{r}) \vec{\zeta}(\vec{r})+\vec{\gamma}(\vec{r})
$$

to comply with the boundary condition $f\left(S_{i}\right)=g\left(S_{o}\right)=1$, $f\left(S_{0}\right)=g\left(S_{i}\right)=0$, and $\vec{\gamma}\left(S_{i}\right)=0$. Then substituting Eq. (5) into Eq. (3) results in a second order partial differential equation:

$$
\begin{aligned}
& \overbrace{\nabla^{2}(f(\vec{r}) \vec{\phi}(\vec{r})+g(\vec{r}) \vec{\zeta}(\vec{r}))}^{\vec{\sigma}}-\overbrace{\omega^{2} \mu \cdot(f(\vec{r}) \vec{\phi}(\vec{r})+g(\vec{r}) \vec{\zeta}(\vec{r}))}^{\omega^{2} \mu \vec{\psi}} \cdot \varepsilon(\vec{r}) \\
& =\nabla[(f(\vec{r}) \vec{\phi}(\vec{r})+g(\vec{r}) \vec{\zeta}(\vec{r})) \cdot \nabla \ln \varepsilon(\vec{r})] .
\end{aligned}
$$
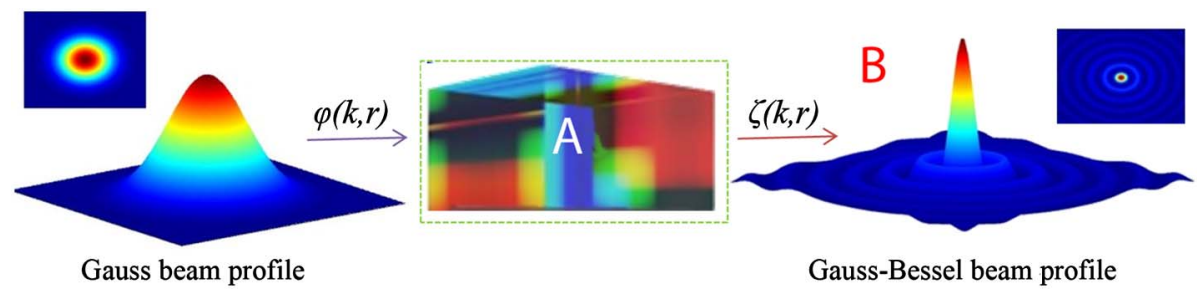

Gauss-Bessel beam profile

Fig. 1. (Color online) Schematic representation of a Gauss to $J_{0}$-Bessel-Gauss transformation via a heterogeneous medium; the transformation device in region $A$ has a refractive index map that needs to be calculated in order to achieve the desired beam transformation. The device is embedded in a homogeneous medium $B$, where Bessel and Gauss beams are known solutions to the Helmholtz equation. 


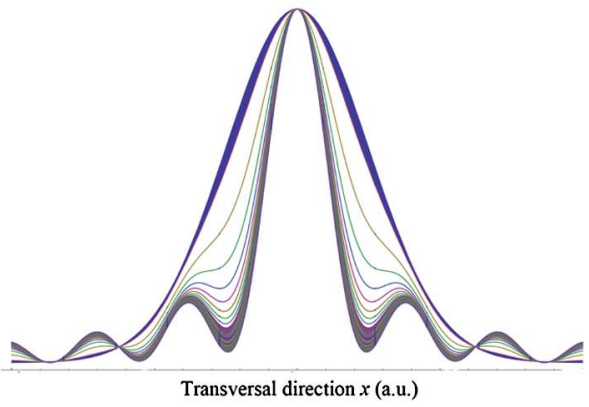

(a) Transformation transition

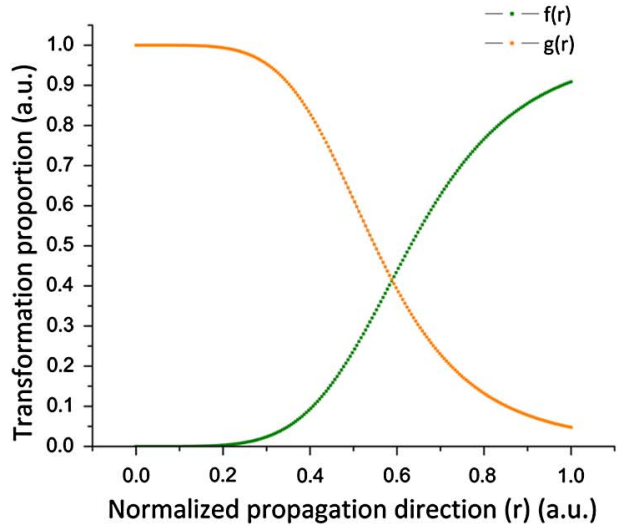

(b) Characteristic functions $f$ and $g$

Fig. 2. (Color online) Illustration of (a) superposition of transition stages in the transformation of Gauss to $J_{0}$-Bessel-Gauss beams, and (b) the transformation functions $f$ and $g$.

Recalling vector calculus identities, the right hand side of Eq. (6) can be reduced to

$$
\begin{aligned}
& \nabla[(f(\vec{r}) \vec{\phi}(\vec{r})+g(\vec{r}) \vec{\zeta}(\vec{r})) \cdot \nabla \ln \varepsilon(\vec{r})] \\
& \quad=(\vec{\psi} \cdot \nabla) \nabla \ln \varepsilon(\vec{r})+\nabla_{\psi}(\nabla \ln \varepsilon(\vec{r}) \cdot \vec{\psi}) .
\end{aligned}
$$

Observe that in Eq. (6), $\vec{\varpi}$ and $\vec{\psi}$ are vector functions with known values across the three domains previously described [see Eq. (4)]. Thus by means of Eq. (7), we can rewrite Eq. (6) as a differential equation that describes the permittivity, $\varepsilon$, as

$$
\omega^{2} \mu \vec{\psi} \varepsilon(\vec{r})+(\vec{\psi} \cdot \nabla) \nabla \ln \varepsilon(\vec{r})+\nabla_{\psi}(\nabla \ln \varepsilon(\vec{r}) \cdot \vec{\psi})=\vec{\varpi}
$$

This is the nonlinear equation for the permittivity that we ought to solve within the boundary conditions and fabrication limitations defined earlier: constant permeability, $\mu$, refractive index range $1.5 \leq n(\vec{r}) \leq 3.5$, where $n(\vec{r})=\sqrt{\varepsilon(\vec{r}) \mu}$, and fabrication minimum feature size $d$.

The computation of an inverse problem as the one hitherto described by Eq. (8) is not trivial, and analytical solutions are rarely found. Moreover, the problem described by the above equation is nonlinear and ill-defined; i.e., its solution is not unique and is dependent continuously on the data [23,24]. As a result, many numerical schemes have been developed to elucidate the solution, v.gr. the adjoint problem [19,24], linear sampling [25-27], variational methods [28], and lately transformation optics [22,29-31]. To find a solution to this quandary, we developed a numerical technique based on the variational method and the adjoint problem.

We begin by noting that if the input and output beams are symmetrical around the propagation axis $z$, and the field in the transformation region is as well, then we can scale down the problem to two dimensions: $z$ and $y$, the longitudinal and transversal direction, respectively. Concerning our matter at hand, both Bessel and $J_{0}$-Bessel-Gauss beams are symmetric around the propagation axis; moreover the transformation field as described by the above equations is also symmetric around the axis $z$. Therefore, our problem can be scaled down to a two dimensional (2D) numerical scheme.

Accordingly, the simulation space is discretized on a grid size $d^{z}$ by $d^{y}$. The solver is given an initial guess of the permittivity map $\varepsilon_{i j}$ across the region $A$ at every point $z_{i}, y_{j}$. The solution to Eq. (3) is computed via a variational approach as described in [28]: at every point $z_{i}$, the transversal function of $\varepsilon_{i j}$ is determined by solving Eq. (ㅇ) to the boundary conditions [see Eq. (5)] $\vec{\psi}\left(y, z_{i 1}\right)$ and $\vec{\psi}\left(y, z_{i+1}\right)$, and the field at that point $\vec{\psi}\left(y, z_{i}\right)$. This iterative approach is carried for every point $z_{i}$. Thus the transversal shape of $\varepsilon_{i j}$, and consequently that of $n_{i j}$, is determined to match the resulting field to the ideal transformation given by Eq. ().

This method does not solely guarantee that the solution will be exempted from extreme or singular values; withal the variational method is especially sensitive to numerical approximations [28]. To account for the latter and work out the extrema in the refractive index, we implement a solution coming from the adjoint problem [24]. The extreme and singular values are approximated to the closest value in the defined range. We then propagate the initial Gauss beam through the device and determine how much the field at every point diverges from the ideal transformation of Eq. (5), including as well the near and far fields. An iterative refinement on this grid regions follows, as described in $[19,24]$, to match the
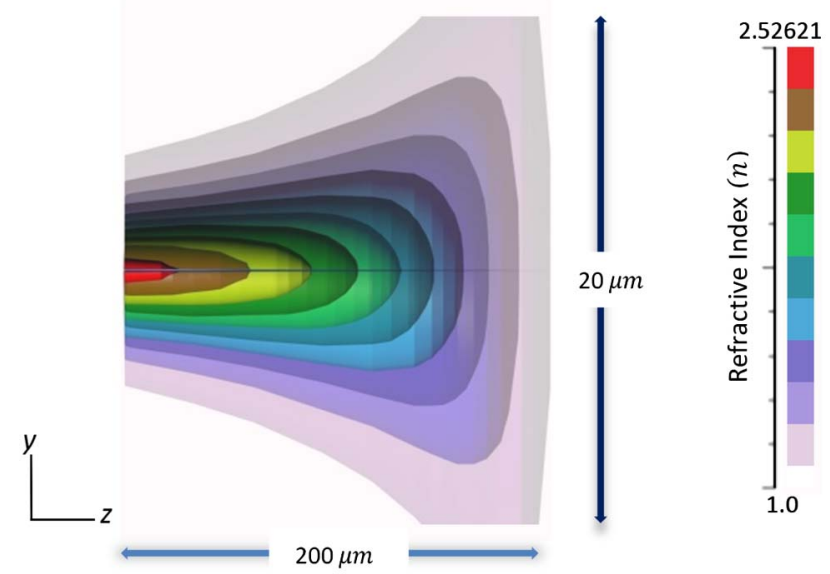

Fig. 3. (Color online) Sketch of the proposed refractive index map for the converter as defined by the transformation functions $f$ and $g$, with a device length of $200 \mu \mathrm{m}$ and a width $25 \mu \mathrm{m}$. Albeit here shown in 11 steps, the refractive index varies smoothly between 1 and 2.57 in controllable steps. The device is rotationally symmetric around the propagation axis $z$. 

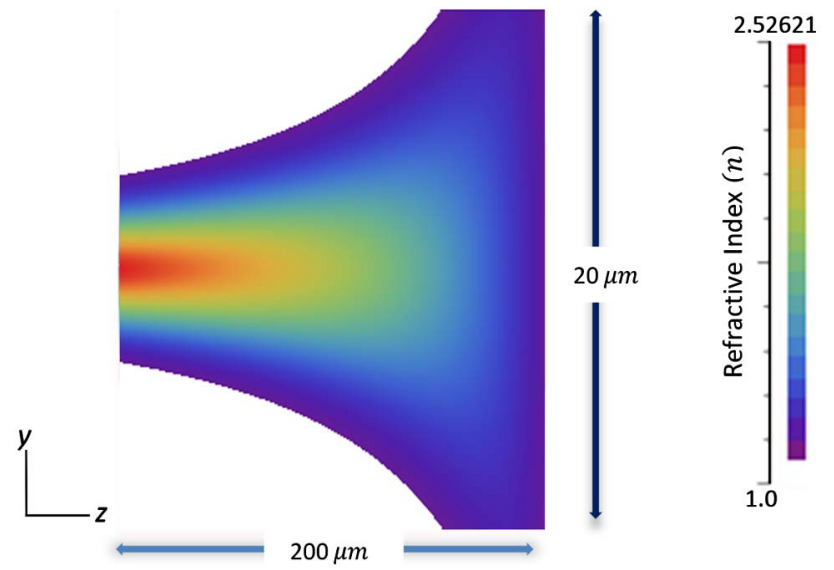

Fig. 4. (Color online) Fine resolution refractive index map for the converter, with minimum feature size $d=1 \mathrm{~nm}$, as defined by the transformation functions $f$ and $g$. Device length is $200 \mu \mathrm{m}$ and width is $25 \mu \mathrm{m}$. The refractive index varies smoothly between 1 and 2.57 in 50 controllable steps. A practical device could be fabricated on a $\mathrm{Si} / \mathrm{Si}_{1 y} \mathrm{O}_{y} / \mathrm{Si}_{1 x y} \mathrm{Ge}_{x} \mathrm{C}_{y}$ slab and the refractive index steps achieved by controlled oxidation or with photo-refractive materials. The device is rotationally symmetric around the propagation axis $z$.

numerical computation output to the ideal $J_{0}$-Bessel-Gauss beam. The beam propagation method is used to propagate the initial Gauss beam through the heterogeneous region $A$.

It is important to note that both the variational and adjoint problem methods, while widely tested, may render inexact solutions due to numerical approximations that can lead to instability and are in general related to the limited resolution of any numerical method. To counterweight this difficulty, the general approach is to use smaller grid sizes even at the expense of computational time increasing exponentially.

\section{RESULTS}

The transformation functions $f$ and $g$ are plotted in Fig. 2(b). The initial guess for the permittivity map is a Gaussian profile in the transversal direction $y$ for all points along $z$, width $\sigma=5$ and a maximum peak of $\varepsilon_{\max }=9$ or $n_{\max }=3$. The initial beam in region $B_{i}$ follows a Gaussian function, with width $10 \mu \mathrm{m}$ and normalized energy to unity.

The outcome of the calculation and optimization process described earlier is depicted in the schematized Fig. 3 and the fine resolution $(d=1 \mathrm{~nm})$ plot of the refractive index in Fig. 4. The refractive index map is symmetric around the propagation axis $z$, and is composed of a smooth variation of the refractive index between 1 and 2.57 . The minimum feature size, $d=d^{z}=d^{y}$, is $1 \mathrm{~nm}$, and has the final dimensions of $200 \mu \mathrm{m}$ long by $20 \mu \mathrm{m}$ wide. Notice that the minimum feature size defines the smoothness of the refractive index map.

The initial impinging Gauss beam in region $B_{i}$ propagates through the device in region $A$, and it is transformed into a $J_{0}$-Bessel-Gauss beam with a $95 \%$ efficiency. Figure 5(a) shows the results for the field at $5 \mu \mathrm{m}$ from the exit of the device, with the corresponding far field depicted in Fig. 5(b). As described earlier, this is the optimized value considering

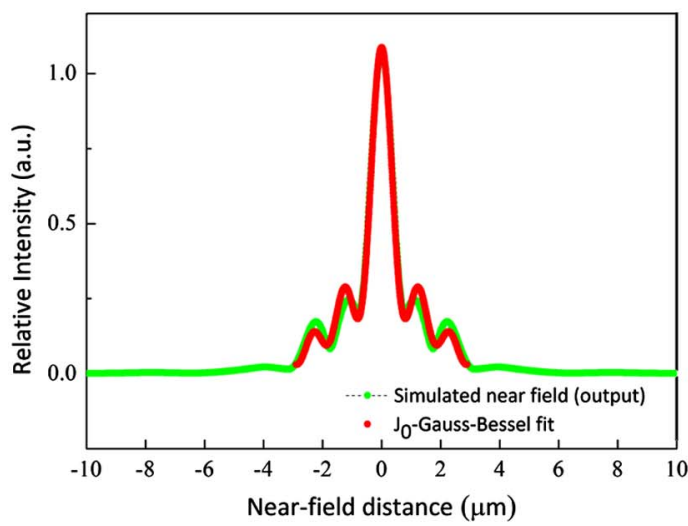

(a)

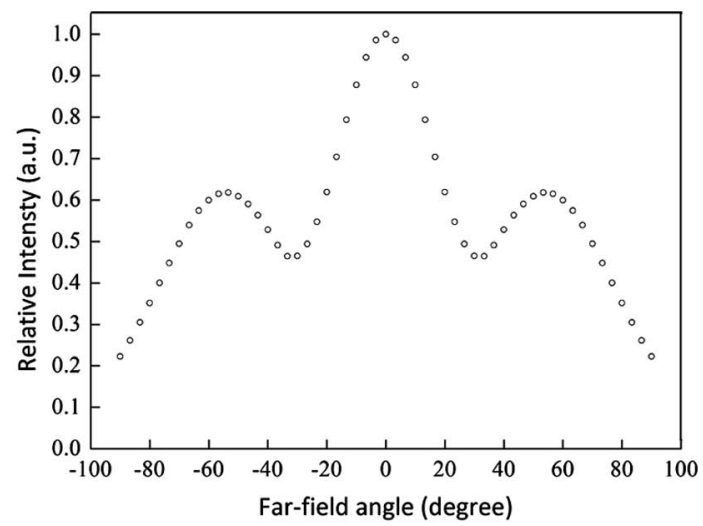

(b)

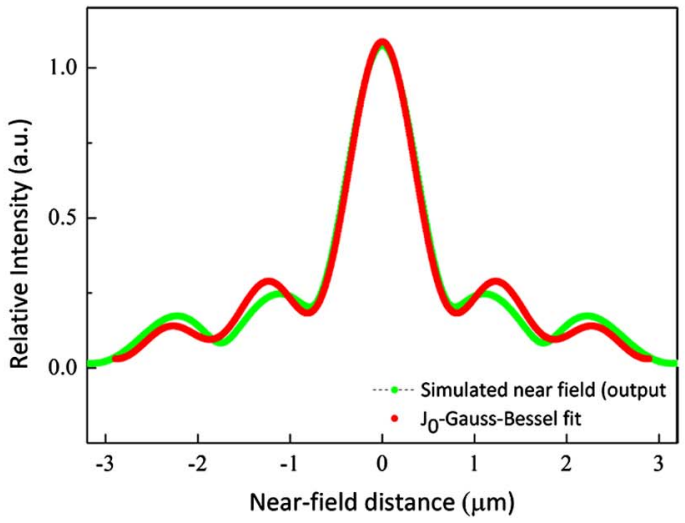

(c)

Fig. 5. (Color online) Transverse electric field intensity of the converted beam: (a) simulated near field computed at $5 \mu \mathrm{m}$ from the output of the device (green), and fit, $95 \%$ confidence, to $J_{0}$-Bessel-Gauss (red). Its far field profile (b), and (c) zoom to principal peak. 
the current state-of-the-art nanofabrication techniques and facilities available to realize the device.

In Fig. 5, it is observed that both near and far fields follow a $J_{0}$-Bessel-Gauss profile with $98 \%$ accuracy. The converted beam divergence is less than $1 \%$ after propagating $50 \mu \mathrm{m}$ and less than $5 \%$ after traveling $1 \mathrm{~mm}$. The energy density at the principal peak is $95 \%$ of the converted beam's total energy, which makes it ideal for OCT applications and free space light-based communication. Losses due to material absorption, scattering, and back reflections are $10 \%$ of the incident field, or $0.457 \mathrm{~dB}$, which can be significantly reduced by using antireflection coatings at the input and output surfaces.

Within the known limitations in fabrication processes, it is noted that not all values of the index of refraction can be achieved. A theoretical study is required to define the tolerance to minimum feature size and its repercussions in the beam profile. We study this case by further modifying the grid size, i.e., the minimum feature size, $d=d^{z}=d^{y}$, from $1 \mathrm{~nm}$ to $10 \mathrm{~nm}$. The results are shown in Fig. $\underline{6}$.

As it is evident from Fig. $6(\mathrm{a})$, modifying the refractive index grid size results in a beam that also resembles a $J_{0}$-BesselGauss beam with a $95 \%$ accuracy, with the sole difference that this beam as it propagates will evolve into a non-Bessel-Gauss beam. This can be drawn from the far field, plotted in Fig. 6(b). Observe that at infinity the field no longer corresponds to the far field of a $J_{0}$-Bessel-Gauss beam. Nevertheless, a coarse mapping will produce a highly focused beam, where $95 \%$ of the energy is localized at the first peak, and the beam diffraction over $1 \mathrm{~mm}$ propagation is less than $5 \%$.
Bessel-Gauss beams, like Bessel beams, exhibit selfregeneration when their path is perturbed by nontransparent scatterers. To examine this self-healing property of the outbound $J_{0}$-Bessel-Gauss beam, we insert a random index medium on its propagation path. The results are shown in Fig. 7 .

The generated $J_{0}$-Bessel-Gauss beam is made to impinge on a random index media, which consists of a circlelike region of $4 \mu \mathrm{m}$, set at $50 \mu \mathrm{m}$ from the output of the device, and a refractive index of $n=2.0$. The scatterer partially obstructs the propagation of the beam. The scattered beam profile is computed at $50 \mu \mathrm{m}$ from the alien obstruction [see Fig. 7(a) and its magnified version in Fig. $7(\mathrm{c})$ ], as well as the far field [see Fig. $7(\mathrm{~b})$ ). As can be seen from the results in Fig. $\underline{7}$, the beam described earlier (see Fig. 5) exhibits self-regeneration. The divergence, computed by comparing the width of the principal peak, from the scattered field to the original transformed beam is $0.3 \%$. Since the beam is not a full Bessel beam, some light is scattered by the object, as expected, giving rise to a loss of $19 \%$ or $0.915 \mathrm{~dB}$.

The 2D mapping for beam conversion heretofore described, while challenging to fabricate, could be built using current nanomanufacturing techniques. A prototype, verbi gratia, could be obtained by controlling the degree of oxidation in $\mathrm{Si} / \mathrm{Si}_{1-y} \mathrm{O}_{y} / \mathrm{Si}_{1-x-y} \mathrm{Ge}_{x} \mathrm{C}_{y}$ [32, $\left.\underline{33}\right]$ systems, or by horizontally stacking nanolayers of photo-refractive materials such as chalcogenide glasses, which have been shown to have a wide range of refractive index [34,35]. The advantages of these materials are that they are relatively well-developed, and the material systems are compatible with PICs and PLCs.

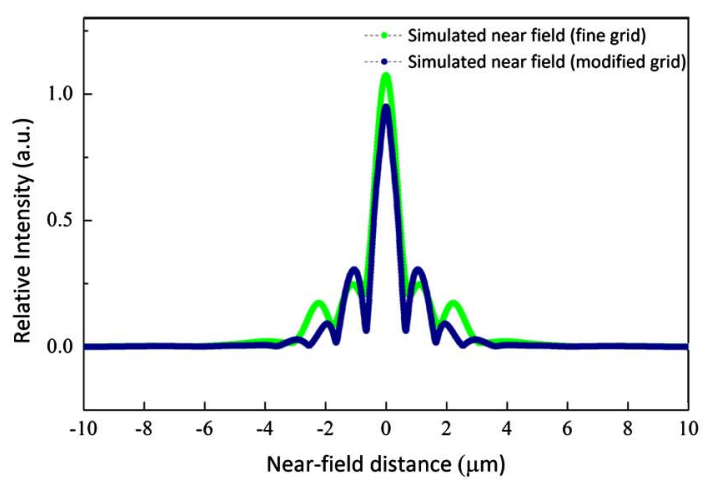

(a)

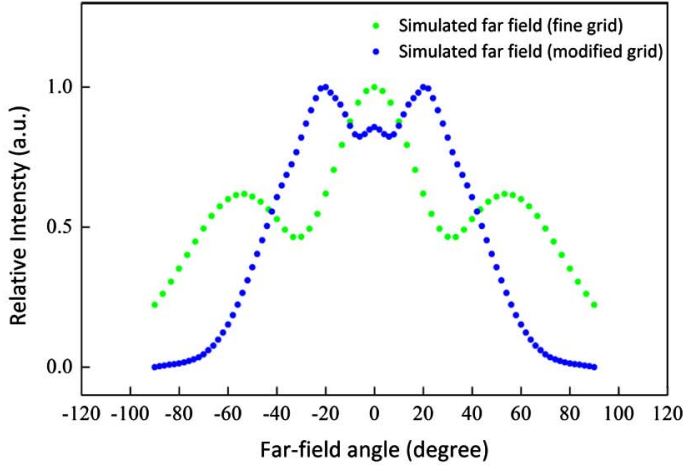

(b)

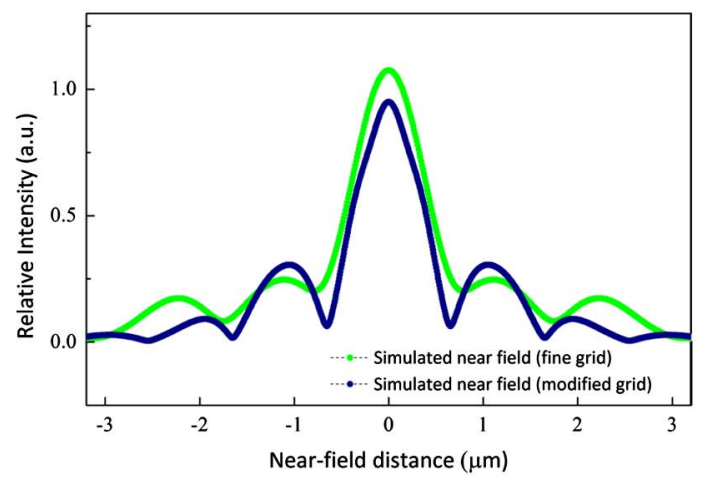

(c)

Fig. 6. (Color online) Transverse electric field intensity profile for the converted beam for two different grid sizes: (a) shows the optimal grid size of $1 \mathrm{~nm}$ (green) and modified grid size of $10 \mathrm{~nm}$ (blue), both computed at the near fields, $5 \mu \mathrm{m}$ from the output of the device. The far fields for both output beams (b), for optimal grid size (green) and modified grid (blue), and (c) magnification of the near field around the principal peak. 


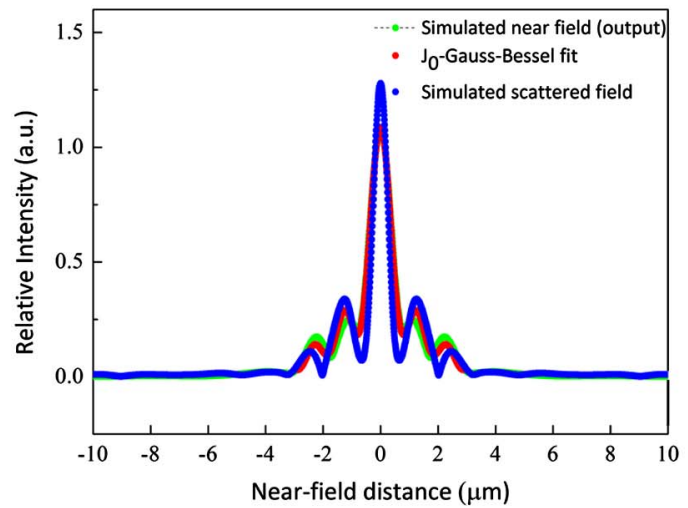

(a)

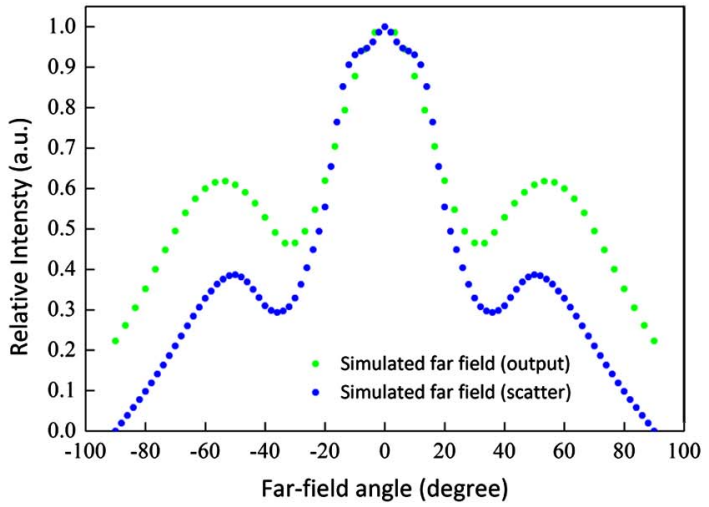

(b)

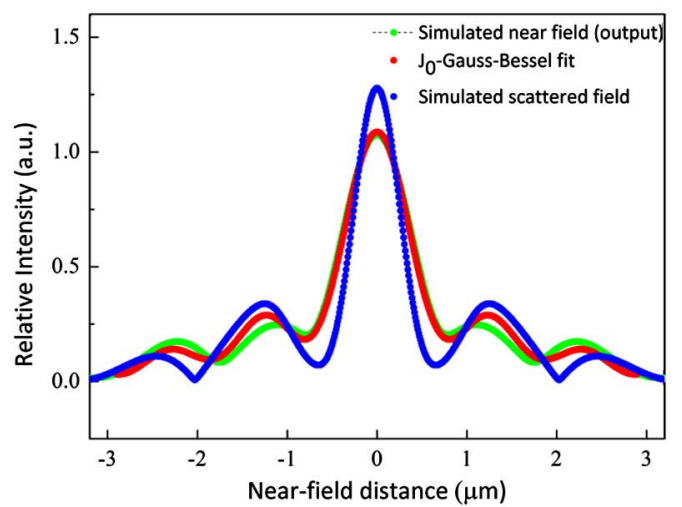

(c)

Fig. 7. (Color online) Transverse electric field intensity profile for (a) the converted beam (green), its fit (red), and the scattered field profile (blue), computed at $50 \mu \mathrm{m}$ from the scattering region. The far field for the undisturbed and perturbed beams are shown in (b), and (c) shows magnification of the fields around the principal peak.

\section{CONCLUSIONS}

We have, to the best of our knowledge, theoretically demonstrated the viability of the first PIC and PLC compatible device utilizing a heterogeneous refractive index map, to achieve the transformation of a Gauss beam into a $J_{0}$-Bessel-Gauss beam. The computed device has a loss of $0.457 \mathrm{~dB}$, and high energy focus, where $95 \%$ of the output beam energy is concentrated at the principal peak of the $J_{0}$-Bessel-Gauss beam profile. The beam has a divergence of $\leq 5 \%$ over a propagation distance of $1 \mathrm{~mm}$, and exhibits self-healing when partially obstructed by an opaque object. The resulting beam has a $J_{0}$-BesselGauss profile for both near and far fields. Note that our design is based on current manufacturing techniques, such as controlled oxidation of $\mathrm{Si} / \mathrm{Si}_{1-y} \mathrm{O}_{y} / \mathrm{Si}_{1-x-y} \mathrm{Ge}_{x} \mathrm{C}_{y}$, or by stacking different layers of chalcogenide glasses. An integrated Gauss to $J_{0}$-Bessel-Gauss beam convertor could enable on-chip applications that take advantage of the beams' self-healing and nondiffractive properties, such as micro optical tweezers, traps and couplers, PICs for telecommunications, and computerized micro tomography and microscopy.

\section{REFERENCES}

1. J. Durnin, "Exact solutions for nondiffracting beams. I. The scalar theory," J. Opt. Soc. Am. A 4, 651-654 (1987).

2. W. Cong, N. Chen, and B. Gu, "Generation of nondiffracting beams by diffractive phase elements," J. Opt. Soc. Am. 15, 2362-2364 (1998).

3. S. Chavez-Cerda, "A new approach to Bessel beams," J. Mod. Opt. 46, 923-930 (1999).
4. F. Gori, G. Guattari, and C. Padovani, "Bessel-Gauss beams," Opt. Commun. 64, 491-495 (1987).

5. Y. Lin, W. Seka, J. H. Eberly, H. Huang, and D. L. Brown, "Experimental investigation of Bessel beam characteristics," Appl. Opt. 31, 2708-2713 (1992).

6. R. M. Herman and T. A. Wiggins, "Propagation and focusing of Bessel-Gauss, generalized Bessel-Gauss and modified BesselGauss beams," J. Opt. Soc. Am. A 18, 170-176 (2001).

7. I. A. Litvin, M. G. McLaren, and A. Forbes, "Propagation of obstructed Bessel and Bessel-Gauss beams," Proc. SPIE 7062, 706218 (2008).

8. J. Durnin, J. Miceli, Jr., and J. H. Eberly, "Diffraction-free beams," Phys. Rev. Lett. 58, 1499-1501 (1987).

9. F. Wu, Y. Chen, and D. Guo, "Nanosecond pulsed Bessel-Gauss beam generated directly from Nd:YAG axicon-based resonator," Appl. Opt. 46, 4943-4947 (2007).

10. V. Arrizón, D. Sánchez-de-la-Llave, U. Ruiz, and G. Méndez, "Efficient generation of an arbitrary nondiffracting Bessel beam employing its phase modulation," Opt. Lett. 34, 1456-1458 (2009).

11. M. M. Méndez Otero, G. C. Martínez Jimnez, M. L. Arroyo Carrasco, M. D. Iturbe Castillo, and E. A. Mart Panameño, "Generation of Bessel-Gauss beams by means of computed generated holograms for Bessel Beams," in Frontiers in Optics, Technical Digest (CD) (Optical Society of America, 2006), paper JWD129.

12. Q. Zhan, "Evanescent Bessel beam generation via surface plasmon resonance excitation by radially polrized beam," Opt. Lett. 31, 1726-1728 (2006).

13. J. Canning, "Diffraction-free mode generation and propagation in optical waveguides," Opt. Commun. 207, 35-39 (2002).

14. V. S. Ilchenko, M. Mohageg, A. A. Savchenkov, A. B. Matsko, and L. Maleki, "Efficient generation of truncated Bessel beams using cylindrical waveguides," Opt. Express 15, 5866-5871 (2007). 
15. T. Tsai, E. McLeod, and C. B. Arnold, "Generating Bessel beams with a tunable acoustic gradient index of refraction lens," Proc. SPIE 6326, 63261F (2006).

16. F. O. Fahrbach, P. Simon, and A. Rohrbach, "Microscopy with self-reconstructing beams," Nat. Photon. 4, 780-785 (2010).

17. M. Lei and B. Yao, "Characteristics of beam profiles of Gaussian beam passing through an axicon," Opt. Commun. 239, 367-372 (2004).

18. K. Hayata, "Are Bessel beams supportable in graded-index media?" Opt. Rev. 3, 299-300 (1996).

19. B. Gang and L. Peijun, "Inverse medium scattering problems for electromagnetic waves," SIAM J. Appl. Math. 65, 2049-2066 (2005).

20. A. J. Devaney, "A filtered backprojection algorithm for diffraction tomography," Ultrason. Imag. 4, 336-350 (1982).

21. R. M. Lewis, "Physical optics inverse diffraction," IEEE Trans. Antennas Propag. 17, 308-314 (1969).

22. Y. Lai, J. Ng, H. Yang, D. Han, J. Xiao, Z. Q. Zhang, and C. T. Chang, "Illusion optics: the optical transformation of an object into another object," Phys. Rev. Lett. 102, 253902 (2009).

23. M. Piana, "On uniqueness for anisotropic inhomogeneous inverse scattering problems," Inverse Probl. 14, 1565-1579 (1998).

24. O. Dorn, H. Bertete-Aguirre, J. G. Berryman, and G. C. Papanicolaou, "A nonlinear inversion method for 3D electromagnetic imaging using adjoint fields," Inverse Probl. 15, 1523-1558 (1999).

25. A. J. Devaney, "A computer simulation study of diffraction tomography," IEEE Trans. Biomed. Eng. BME-30, 377-386 (1983).
26. H. Hadar and P. Monk, "The linear sampling method for solving the electromagnetic inverse medium problem," Inverse Probl. 18, 891-906 (2002).

27. H. Hadar, "The interior transmission problem for anisotropic Maxwell's equations and its applications to the inverse problem," Math. Methods Appl. Sci. 27, 2111-2129 (2004).

28. F. Cakoni, "A variational approach for the solution of the electromagnetic interior transmission problem for anisotropic media," Inverse Probl. Imaging 1, 443-456 (2007).

29. J. B. Pendry, D. Schurig, and D. R. Smith, "Controlling electromagnetic fields," Science 312, 1780-1782 (2006).

30. U. Leonhardt and T. G. Philbin, "Transformation optics and the geometry of light," Prog. Opt. 53, 69-152 (2008)

31. H. Chen, C. T. Chan, and P. Sheng, "Transformation optics and metamaterials," Nat. Mater. 9, 387-396 (2010).

32. H. J. Lee, C. H. Henry, K. J. Orlowsky, R. F. Kazarinov, and T. Y. Kometani, "Refractive-index dispersion of phospho-silicate glass, thermal oxide, and silicon nitride films on silicon," Appl. Opt. 27, 4104-4109 (1988).

33. W. Fenga, W. K. Choia, L. K. Beraa, M. Jib, and C. Y. Yangb, "Optical characterization of as-prepared and rapid thermal oxidized partially strain compensated $\mathrm{Si}_{1 x y} \mathrm{Ge}_{x} \mathrm{C}_{y}$ films," Mater. Sci. Semicond. Process. 4, 655-659 (2001).

34. A. Zakery, "Optical properties and applications of chalcogenide glasses: a review,” J. Non-Cryst. Solids 330, 1-12 (2003).

35. S. B. Kang, "Optical and dielectric properties of chalcogenide glasses at terahertz frequencies," ETRI J. 31, 667-674 (2009). 